Published in final edited form as:

Cell Rep Phys Sci. 2021 September 22; 2(9): . doi:10.1016/j.xcrp.2021.100552.

\title{
A self-degradable hydrogel sensor for a nerve agent tabun surrogate through a self-propagating cascade
}

\author{
Doo-Hee Lee ${ }^{1}$, Stephanie A. Valenzuela ${ }^{1}$, Manuel N. Dominguez ${ }^{1}$, Mai Otsuka², Delia J. \\ Milliron $1,3,{ }^{*}$, Eric V. Anslyn ${ }^{1,4,{ }^{*}}$ \\ ${ }^{1}$ Department of Chemistry, University of Texas at Austin, Austin, TX 78712, USA \\ ${ }^{2}$ National Research Institute of Police Science, 6-3-1 Kashiwanoha, Kashiwa, Chiba, 277-0882, \\ Japan
}

${ }^{3}$ McKetta Department of Chemical Engineering, University of Texas at Austin, Austin, TX 78712, USA

${ }^{4}$ Lead contact

\section{SUMMARY}

Nerve agents that irreversibly deactivate the enzyme acetylcholinesterase are extremely toxic weapons of mass destruction. Thus, developing methods to detect these lethal agents is important. To create an optical sensor for a surrogate of the nerve agent tabun, as well as a physical barrier that dissolves in response to this analyte, we devise a network hydrogel that decomposes via a self-propagating cascade. A Meldrums acid-derived linker is incorporated into a hydrogel that undergoes a declick reaction in response to thiols, thereby breaking network connections, which releases more thiols, propagating the response throughout the gel. A combination of chemical reactions triggered by the addition of the tabun mimic initiates the cascade. The dissolving barrier is used to release dyes, as well as nanocrystals that undergo a spontaneous aggregation. Thus, this sensing system for tabun generates a physical response and the delivery of chemical agents in response to an initial trigger.

\section{Graphical Abstract}

\footnotetext{
This is an open access article under the CC BY-NC-ND license (http://creativecommons.org/licenses/by-nc-nd/4.0/).

*Correspondence: milliron@che.utexas.edu (D.J.M.), anslyn@austin.utexas.edu (E.V.A.). AUTHOR CONTRIBUTIONS

E.V.A. and D.-H.L. conceived the project, designed all experiments, and wrote the manuscript. D.-H.L. synthesized hydrogels and all organic materials except ligands for NCs and carried out all experiments excluding optimizing the conditions. S.A.V. synthesized ligands for the NCs and edited the manuscript. M.N.D. prepared the NCs and recorded the scanning transmission electron microscopy (STEM) image data. M.O. optimized the conditions. D.J.M. revised the manuscript.

SUPPLEMENTAL INFORMATION

Supplemental information can be found online at https://doi.org/10.1016/j.xcrp.2021.100552.

DECLARATION OF INTERESTS

The authors declare no competing interests.
} 


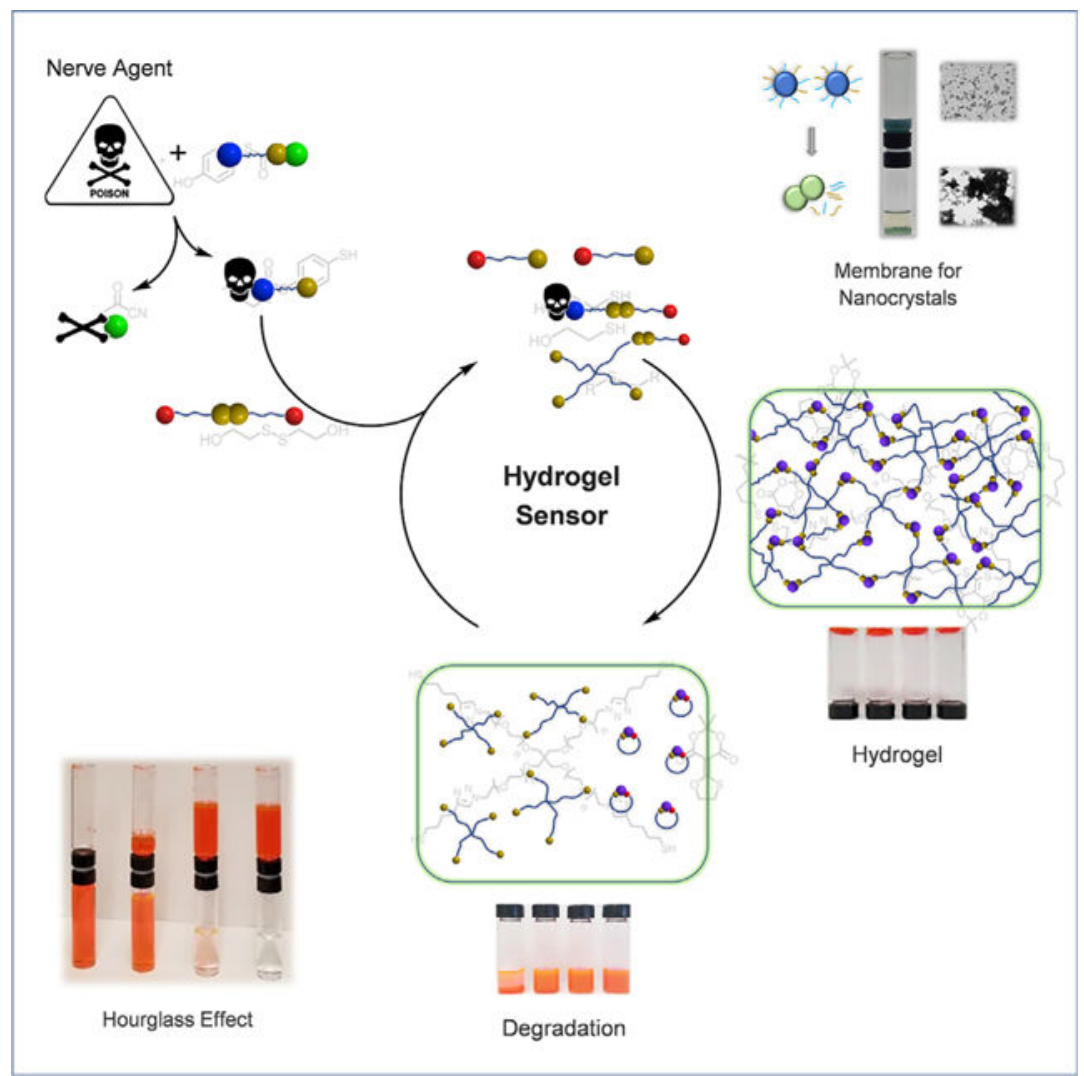

Lee et al. describe a self-degradable hydrogel that is used as both an optical and physical-change sensor for a nerve agent tabun surrogate. Upon detection, the hydrogel will degrade through a self-propagating cascade. Furthermore, the hydrogel acts as a barrier in an hourglass, as is confirmed using nanocrystals.

\section{INTRODUCTION}

A hydrogel is a network in which a hydrophilic polymer forms a three-dimensional architecture that gels water. Hydrogels do not dissolve in an aqueous environment; instead they swell and contain large amounts of water. ${ }^{1,2}$ Because of these characteristics, hydrogels are flexible enough to act like natural tissues. In addition, it is feasible to control the mechanical integrity, porosity, and degradation properties of hydrogels by adjusting parameters such as synthesis conditions, types of polymers used in the gel, molecular weight (MW), and crosslinking density. As a result, hydrogels are widely used in biomedical applications such as contact lenses, hygiene products, wound dressings, scaffolds for protein recombination, and drug delivery systems. ${ }^{3,4}$

Hydrogels applied to delivery systems are used to load and release drugs by exploiting their porous structure and/or degradation. ${ }^{3,5}$ Their porosity can be changed by controlling the crosslinking density as well as the polymer's affinity for water in the matrix. One can load a drug by mixing it with a precursor of the hydrogel or by swelling the hydrogel in a solution of the drug. The release proceeds through various mechanisms such as diffusion control, 
swelling control, chemical control, and environment-reactive release. ${ }^{6}$ Therefore, the loading and degradation ability of hydrogels is a critical factor in delivery systems. We envisioned that environment-reactive release could be used both in the detection of and for triggering a response to chemical warfare agents (CWAs).

CWAs are highly hazardous compounds that have been used in terrorism as well as in wars. Among CWAs, nerve agents that contain an organophosphorus functional group have been the most exploited weaponized agent. They trigger activation of the parasympathetic nervous system, causing fatal injuries to humans. Since first developed during World War II, nerve agents have been used in the Iraq-Iran conflict from 1980 to $1988,{ }^{7}$ the Syrian civil war in 2013, an assassination in 2017 of a North Korean political affiliate, and the death of a former Russian military officer in the United Kingdom in 2018. Even though CWAs were banned by the Organisation for the Prohibition of Chemical Weapons (OPCW), and 193 member states joined the convention, ${ }^{8}$ they are still used because of their low price and ease of synthesis. To avoid these threats, it is important to detect and eliminate them.

Hydrogels have been used previously in nerve agent detection, including studies of the release of entities attached to the gels, ${ }^{9,10}$ fluorescence sensors using fluorophore-integrated smart hydrogels, ${ }^{11}$ and photonic crystal hydrogel sensors. ${ }^{12,13}$ However, these studies induced small changes in the signaling units included in the hydrogel or the particle spacing.

Herein, we report a hydrogel that self-degrades via a self-propagating cascade in response to a surrogate of the nerve agent tabun. The hydrogel undergoes a large physical change (i.e., complete degradation) when triggered to do so by a chemical agent generated from the nerve agent mimic, thereby transforming the hydrogel from a gel to a sol easily seen with the naked eye. Furthermore, because this reaction is carried out in a self-propagating cascade, less than an equivalent of the trigger is needed to induce dissolution. The self-propagating cascade amplifies the detection through a cascade cycle, so only parts-per-billion (ppb) levels of analyte are needed. ${ }^{14}$ Furthermore, this sensor was designed to detect the tabun surrogate selectively among a variety of nerve agents. Because only tabun has a cyanide as a leaving group, a series of reactions initiated by cyanide that generate a thiol trigger was developed, thereby imparting selectivity to the sensing event.

\section{RESULTS AND DISCUSSION}

\section{Design criteria}

We previously reported that derivatives of Meldrum's acid, 1, shown in Scheme 1, can click together different thiols, or amines with thiols, following by declicking with $\beta$-mercaptoethanol (BME) or dithiothreitol (DTT), respectively. ${ }^{15}$ In the context of an autoinductive cascade, such a monomer can be used to amplify the number of thiols present in a solution via addition of ppb levels of a thiol trigger. ${ }^{14}$ As Scheme 1 portrays, addition of the trigger to a solution of disulfide-linked BME (BMEox) leads to disulfide exchange, releasing BME that declicks $\mathbf{1}$ to release two equivalents of methanethiol and product $\mathbf{2}$, thus doubling the number of thiol triggers each time the cycle turns over until all of $\mathbf{1}$ is consumed. This cascade was previously used to create an exquisitely sensitive sensor for V-nerve agent surrogates because thiols are the biproducts of their hydrolysis. ${ }^{14}$ 
Tabun, however, is not a V-nerve agent and thus does not release thiols but instead releases cyanide upon hydrolysis. ${ }^{16}$ Thus, in order to trigger the auto-inductive cascade of Scheme 1 , we required a series of reactions that could occur with a tabun mimic to ultimately generate an equivalent of thiol for each equivalent of the mimic. Because of the risks associated with working with nerve agents, experiments are generally performed using surrogate molecules that are safer to handle than real CWAs but with similar chemical behavior and structure. ${ }^{17-20}$ We choose diethyl cyanophosphonate (DCNP) as the tabun mimic, and as described below, two reactions initiated by cyanide to release a thiol were devised (Scheme 2). Admittedly, for the real agent the experimental conditions may need to be adjusted to accommodate small differences in reactivity.

We have also previously reported that Meldrum's acid derivatives analogous to $\mathbf{1}$ can be used as linkers to generate hydrogels using thiol containing monomers and that the gels can be degraded to small molecules with equal equivalents of BME or DTT to the Meldrum's acid linker. ${ }^{21}$ In this work, hydrogel 3, shown in Scheme 2, was prepared via copper-catalyzed azide-alkyne cycloaddition $(\mathrm{CuAAC})$ reactions to introduce branched monomers (PEG-azide; MW = 10,000) and create triazole linkers (blue), while the Meldrum's acid linkers (green) were used to degrade in response to the addition of BME or DTT. Similarly, the Kalow group has recently reported a series of polymers using analogous linking units. They developed vitrimeric silicone elastomers with a Meldrum's acid derivative and thiol-functionalized polydimethylsiloxane through a conjugate addition of thiols. Adding 1-mercapto-2-propanol to the expanded network permanently disrupted the network crosslinking, resulting in complete dissolution. ${ }^{22}$

The goal of the study presented here was to combine the auto-inductive cascade of Scheme 1 with the degradable hydrogel shown in Scheme 2. The hypothesis was that BME declick of any of the Meldrum's acid-derived linkages in $\mathbf{3}$ would result in two equivalents of thiol being generated, analogous to the two equivalents of methanethiol generated in the cascade shown in Scheme 1. Thus, the gel generates the thiols that propagate the cycle as the degradation proceeds, each time creating the fivemembered ring product 2 (Scheme 3 ). In essence, the gel self-degrades upon being triggered with a thiol. Finally, we envisioned that triggering the auto-inductive degradation of a physical barrier created by the self-degrading hydrogel could be used to release chemical agents, and we demonstrate this with both colorimetric indicators and nanocrystals (NCs).

\section{Preliminary test}

In our previous work, compound $\mathbf{1}$ was successfully used in the auto-inductive cascade shown in Scheme 1 using butanethiol as the trigger in a $\mathrm{pH} 10$ buffered solution (borax/sodium hydroxide buffer). ${ }^{14}$ Before carrying out the detection of a tabun mimic, a preliminary test was conducted to determine if placement of Meldrum's acid linkers analogous to $\mathbf{1}$ within gel $\mathbf{3}$ would lead to a similar cascade (Scheme S1; Figure S1).

Rhodamine 6-G (R6G) was added during the synthesis of hydrogel 3 to confirm the changes with a quantitative analysis. Kinetics experiments were conducted with the R6G-containing gel, 2-hydroxyethyl disulfide (BMEox), and butanethiol at different concentrations. The equivalents were determined relative to the number of the Meldrum's acid linking units in 3. 
Each compound was added to centrifuge tubes with $5 \mathrm{~mL}$ of $\mathrm{pH} 10$ buffer (see Supplemental information for details). After shaking the tubes for 5, 10, 20, 30, 40, 50, and $60 \mathrm{~min}, 100$ $\mu \mathrm{L}$ of solution was collected in a pipette for optical analysis. According to these time points, gel 3 was entirely degraded after $40 \mathrm{~min}$ in both 0.1 and 0.3 equivalents of butanethiol, whereas the changes in the two other samples at $\mathrm{pH} 10$, without the thiol trigger, showed only $15 \%-20 \%$ as much R6G release (Figures S1-S3), presumably because of some extent of gel degradation or diffusion of R6G out of the hydrogel. Another key difference with the study reported here is the use of $\mathrm{pH} \mathrm{7.3,} \mathrm{which} \mathrm{nearly} \mathrm{eliminates} \mathrm{all} \mathrm{degradation} \mathrm{and}$ R6G release without the addition of a thiol (see Figure 2). Thus, this study confirmed that if a substance capable of reducing BMEox was introduced to the gel, ${ }^{23}$ the auto-inductive cascade would be triggered to commence and the gel thereby entirely self-degraded.

\section{Optimized conditions and rate constant for the thiol trigger}

To generate a thiol trigger for detection of tabun surrogate DCNP, initiator 4 was designed (Scheme 3). We anticipated that the phenol group of $\mathbf{4}$ would add to the phosphoryl center of DCNP and the thioester would be electrophilic enough to capture the cyanide leaving group, thereby generating aromatic thiol 5 that could be used to trigger the dissolution of gel 3. Compound 4 is both nucleophilic (the phenol) and electrophilic (the thioester), yet it is a stable white powder and does not react with itself in solution at $\mathrm{pH}$ 7. However, as previously stated, to generate product 5 the phenol must react with DCNP, ${ }^{24,25}$ while the thioester must react with cyanide. ${ }^{26}$ Thus, we sought to explore the relative rates of these two reactions that involve 4 . We first varied four conditions to maximize the yield of $\mathbf{5}$ : solvent, temperature, time, and basicity. Acetonitrile, acetone, DMF, DMSO, and water were tested as solvents. Among them, acetonitrile had the highest yield of 5. Similarly, we varied temperature, time, and basicity. As a result, an optimized condition for the highest yield of $\mathbf{5}$ was obtained: $25^{\circ} \mathrm{C}, 30 \mathrm{~min}$, and 2 equivalents of triethyl amine (TEA) in acetonitrile (Table $\mathrm{S} 1)$.

Using these conditions, we set out to explore model reactions to analyze the relative rates of the reactions that would initiate the cascade of Figure 1. There were two model reactions. The first was a model of the phenol attacking the phosphorus center of DCNP (Figure $1 \mathrm{~A}$ ), and the second was a model for the released cyanide reacting with a thioester (Figure 1B). In 6 the thioester of initiator 4 was replaced with a thioether, and in 7 the phenol of $\mathbf{4}$ was protected with a methyl group. The kinetic studies were carried out via UV-Vis spectroscopy. While keeping the concentration of $\mathbf{6}$ and $\mathbf{7}$ constant, the change of the reaction was followed for $2 \mathrm{~h}$ with different DCNP and cyanide concentrations (1, 2, 3, and 4 equivalents), respectively (Figures S4 and S6). Also, the kinetic studies between DCNP and 6 were conducted using ${ }^{1} \mathrm{H}$ nuclear magnetic resonance (NMR) (Figure S5). For the reaction of cyanide anion with 7, we used 18-crown-6 $(50 \mu \mathrm{M})$ to dissolve potassium cyanide $(50 \mu \mathrm{M})$. The concentrations of 6 and 7 were monitored, and using these results, rate constants were calculated. ${ }^{27}$ The second-order rate constant of the reaction between 6 and DCNP was $0.66 \mathrm{M}^{-1} \mathrm{~min}^{-1}$ (Figure S4D), and for the reaction between 7 and cyanide anion the rate constant was $2.67 \mathrm{M}^{-1} \mathrm{~min}^{-1}$ (Figure S6D). Thus, the reaction of the phenol with DCNP is approximately 4 times slower than the reaction of cyanide with the thioester. 
Hence, it is the reaction between the trigger and the tabun surrogate that limits the rate of the initiator 4 to trigger the cascade.

\section{Titration studies at $\mathrm{pH} \mathbf{1 0}$ and 7.3}

Once the conditions for the reactions that would initiate the auto-inductive cascade and their model kinetics were obtained, we turned to triggering the dissolution of hydrogel 3. After the initiation step was conducted in acetonitrile to generate the thiol trigger, aliquots were transferred to aqueous solutions containing the gels, and the cascade studies were performed in $\mathrm{pH} 10$ buffer (as were the preliminary tests using butanethiol described above). There were four samples of equal weight of gel $\mathbf{3}(128 \mathrm{mg})$ and equal concentrations of probe 4 $(300 \mu \mathrm{M})$, while the amount of DCNP was varied between $0,0.05,0.1$, and 0.3 equivalents relative to the Meldrum's acid linkers in hydrogel 3. As anticipated, the three samples containing DCNP led to hydrogel degradation (Figures S7-S9), while 0 equivalents gave far less degradation. This result is meaningful because it confirmed that probe 4 released a thiophenol that in turn triggered the dissolution of the hydrogel.

We next explored physiological $\mathrm{pH} 7.3$ instead of $\mathrm{pH} 10$, all other conditions remaining the same. At pH 7.3, thiophenols (such as $\mathbf{5}$ ) are substantially ionized, while alkylthiols (such as butanethiol; recall Figures S1-S3) are not, ${ }^{28}$ and hence we expected the cascade to still function well at this lower $\mathrm{pH}$. Indeed, the results were similar except that the hydrogel took longer to degrade (time-dependent data; Figure 2C). The shape of the time-dependent data plots shows an initiation period as the cascade starts degrading, an exponential rise due to auto-induction, followed by a plateau as all the Meldrum's acid linkers in $\mathbf{3}$ are consumed. In the absence of DCNP, 3 was hardly degraded and after 2-3 days it remained undegraded, although loosened. Furthermore, at either $\mathrm{pH} 10$ or 7.3, the Meldrum's acid linkers in gel 3 could be observed to generate $\mathbf{2}$ via UV-Vis absorption spectroscopy due to a hypsochromic shift of the $\lambda_{\max }$ of $350 \mathrm{~nm} \rightarrow 290 \mathrm{~nm}$ (Figure 2D), as we have observed in previous systems. ${ }^{14}$ Hence, Figure 2 shows three different ways to follow the optical response to the tabun mimic DCNP. Although these three responses could be used to quantitate the concentration of DCNP as we have done in other contexts, ${ }^{14}$ the goal for this study was to trigger gel degradation and use it as a delivery agent. Interestingly, however, there is another informative aspect to the UV-Vis traces given in Figure 2D that gives insight into the time evolution of gel degradation. As the peak at $350 \mathrm{~nm}$ increased and decreased according to the time sequence, the peak at $290 \mathrm{~nm}$ indicative of $\mathbf{2}$ continually increased. Initially, because hydrogel $\mathbf{3}$ was in a solid state, the solution did not show its absorbance. However, as $\mathbf{3}$ gradually degraded, some of gel with the Meldrum's acid linker (a chromophore itself) dissolved and was observed at $350 \mathrm{~nm}$. As the cascade continued the Meldrum's acid linker entirely broke down to product $\mathbf{2}$, thereby continuing to increase the peak at $290 \mathrm{~nm}$.

\section{Selectivity test}

After confirming that this hydrogel sensor was working properly for DCNP, a selectivity experiment was performed. A unique characteristic of tabun is the cyanide moiety, which acts as a leaving group and a nucleophile. The Kumar group has reported the analysis of cyanide due to its nucleophilic ability after being released from an analogous mimic. ${ }^{29-31}$ In our study, the two reactions that use initiator $\mathbf{4}$ were anticipated to impart selectivity to 
the sensing event. To test this, we conducted experiments on three nerve agent surrogates (Figures S10-S12). First, the reaction between the initiator 4 with the nerve mimics DCNP (a GA-agent mimic), diisopropylfluorophosphate (DFP; a GB/GD-agent mimic), and demeton-S-methyl (DSM; a V-agent mimic) were followed by liquid chromatography-mass spectrometry (LC-MS). The thiophenol compound $\mathbf{5}$ was observed only in the reaction with DCNP. In the reaction of $\mathbf{4}$ with DFP, phosphoryl transfer to the phenol did occur, but the thioacetate remained intact. With DSM, there was no reaction with initiator 4 . We also attempted to initiate the auto-inductive cascade to degrade gel $\mathbf{3}$ using the three nerve agent mimics. As expected, the hydrogel was degraded only when DCNP was added, and the absorption increased compared with DFP and DSM. Similarly, in a study by the Martínez-Máñez group, it was reported that when comparing DFP and DCNP, only DCNP reacted with both an aldehyde and phenol moiety within their probe. ${ }^{32}$

\section{Membrane degradation}

We next turned to using hydrogel $\mathbf{3}$ as a physical barrier to time the release of reagents in response to DCNP using an "hourglass"-like study. Although in a real-life application the reagents could be tabun antidotes or capture agents, we mimicked such entities using dyes and NCs, respectively. We anticipated that solutions resulting from the reaction of 4 and DCNP could be used to trigger degradation of a membrane created from 3 in a time-dependent manner depending upon the thickness of the membrane. Hydrogels of four different thicknesses were prepared and affixed to vial adaptors. To do so, the masses of reactants and quantities of solvent ( $t$-butanol and water) were controlled to make different thickness of membranes (Figure S13). Indeed, we visually confirmed that the membrane decomposition time varies contingent on the thickness of the hydrogel (Figure 3) because the thicker the membranes, the longer it took for the gels to dissolve and release the red solution containing R6G into the bottom vial. Several factors must influence this phenomenon, each of which could be tuned for a specific application: (1) the degradation rate of the hydrogel induced by DCNP is dependent upon $\mathrm{pH},(2)$ the weight of the top solution, and (3) the surface tension of the solvent (water in our case) that impedes the solution from passing through the membrane.

Furthermore, we anticipated that the membrane could be used to release particles, such as potentially capture agents. To study this, we followed the change in morphology of $\mathrm{NC}$ particles upon mixing solutions because they give an optical response. We found that $\mathrm{Sn}: \mathrm{In}_{2} \mathrm{O}_{3} \mathrm{NCs}$ (ITO NCs) functionalized with mixed aldehyde and benzyl-terminal oligo(ethylene oxide) ligands can be dispersed in polar solvents. ${ }^{33}$ Specifically, we found that these functionalized ITO NCs form stable dispersions in aqueous solutions above $\mathrm{pH} 8$, but aggregate at low $\mathrm{pH}$. Thus, to explore the ability to release particles, we followed the $\mathrm{pH}$-controlled aggregation using the self-degradable hydrogel. The top vial was filled with a dispersion of 12-nm-diameter $\mathrm{NCs}(\mathrm{pH} 10)$, which was triggered to fall into the bottom vial $(\mathrm{pH} \sim 3)$ using the same procedure as described in the previous paragraph. Once the membrane dissolved and the NC solution mixed with the acidic water, they flocculated out of solution. We believe this occurs from a destabilized ligand shell from ligand exchange/ removal and etching conditions created by the use of the strong acid (Figure 4A) ${ }^{34,35}$ The blue color of the NCs in the top vial is due to the high-energy tail of the localized surface 
plasmon resonance (LSPR) absorbing red light, while the aggregated NCs (where strong coupling of LSPR in neighboring NCs is expected) appear light green in color (Figures 4B4D). Thus, physical changes of NCs can also be used to monitor/sense the gel dissolution response to the tabun surrogate.

\section{Conclusions}

A degradable hydrogel was used as both an optical and physical-change sensor for a surrogate of the nerve agent tabun. Using a self-propagating cascade that degrades the gel due to the release of thiols that then further propagates its own degradation, we could monitor absorbance changes, dye release, and physical changes of the gel. The initiation of the cascade was performed via two sequential reactions using an initiator that reacts with the tabun surrogate, releases cyanide, and subsequently releases a thiophenol to trigger gel degradation. Confirmation of the anticipated two reaction sequence was done by monitoring the kinetics of model reactions. In addition, the hydrogel was used as a membrane to study the decomposition rate depending on the membrane thickness, thus generating an hourglass-like change in the mixing of solutions of colored dyes and NCs that undergo dispersion changes, although one can imagine the delivery of other chemical agents upon triggering with tabun or surrogates. For a practical application with a real agent, the kinetics of this proof-of-principle system will need to be sped up, because an hour or more response time is too slow to be practical. Thus, on another front we are working on more rapid self-propagating cascades that can be incorporated into gels. Furthermore, the dissolution rate of the gel has the potential to act as a quantitative assay, which will be further explored in the future. Last, a more practical approach would avoid our two-step protocol involving incubation of the surrogate with the initiator, followed by addition to the gel. Importantly, on the basis of our results, we expect that the concept of triggerable self-degradable hydrogels can also be applied in other fields, such as bio- and nanomaterials.

\section{EXPERIMENTAL PROCEDURES}

\section{Resource availability}

Lead contact-Further information and requests for resources should be directed to and will be fulfilled by the lead contact, Eric V. Anslyn (anslyn@austin.utexas.edu).

Materials availability—This study did not generate new materials.

Data and code availability-The published article includes all datasets generated or analyzed during this study.

\section{Synthetic procedures and characterization}

See Supplemental experimental procedures, Figures S1-S31, Scheme S1, and Table S1.

\section{Supplementary Material}

Refer to Web version on PubMed Central for supplementary material. 


\section{ACKNOWLEDGMENTS}

This work was supported primarily by the National Science Foundation (NSF) through the Center for Dynamics and Control of Materials: an NSF Materials Research Science and Engineering Center (MRSEC) under Cooperative Agreement DMR-1720595 and NMR instruments obtained through NIH grant 1 S10 OD021508-01. For this project, S.A.V. received funding from the NSF Graduate Research Fellowship Program (DGE-1610403). Additional funding for this project came from the Welch Foundation (F-1848 and F-1696). E.V.A. acknowledges support from the Welch Regents Chair (F-0046). D.-H.L. acknowledges support from ROKA. M.O. received support from the Japan Society for the Promotion of Science (JSPS) Overseas Challenge Program for Young Researchers.

\section{REFERENCES}

1. Thakur S, Thakur VK, and Arotiba OA (2018). History, classification, properties and application of hydrogels: an overview. In Hydrogels, Thakur VK and Thakur MK, eds. (Springer), pp. 29-50.

2. Bahram M, Mohseni N, and Moghtader M (2016). An introduction to hydrogels and some recent applications. In Emerging Concepts in Analysis and Applications of Hydrogels, Majee SB, ed. (IntechOpen), pp. 9-38.

3. Calo E, and Khutoryanskiy VV (2015). Biomedical applications of hydrogels: a review of patents and commercial products. Eur. Polym. J 65, 252-267.

4. Ozcelik B (2016). Degradable hydrogel systems for biomedical applications. In Biosynthetic Polymers for Medical Applications, Poole-Warren L, Martens P, and Green R, eds. (Woodhead Publishing), pp. 173-188.

5. Ashley GW, Henise J, Reid R, and Santi DV (2013). Hydrogel drug delivery system with predictable and tunable drug release and degradation rates. Proc. Natl. Acad. Sci. U S A 110, 2318-2323. [PubMed: 23345437]

6. Kim SW, Bae YH, and Okano T (1992). Hydrogels: swelling, drug loading, and release. Pharm. Res 9, 283-290. [PubMed: 1614957]

7. Stuart JA, Ursano RJ, Fullerton CS, Norwood AE, and Murray K (2003). Belief in exposure to terrorist agents: reported exposure to nerve or mustard gas by Gulf War veterans. J. Nerv. Ment. Dis 191, 431-436. [PubMed: 12891089]

8. Organisation for the Prohibition of Chemical Weapons (OPCW) (2021). Home page. https:// www.opcw.org/.

9. Belger C, Weis JG, Egap E, and Swager TM (2015). Colorimetric stimuli-responsive hydrogel polymers for the detection of nerve agent surrogates. Macromolecules 48, 7990-7994.

10. Ali MA, Tsai T-H, and Braun PV (2018). Amplified detection of chemical warfare agents using two-dimensional chemical potential gradients. ACS Omega 3, 14665-14670. [PubMed: 30555985]

11. Whitaker CM, Derouin EE, O'connor MB, Whitaker CK, Whitaker JA, Snyder JJ, Kaufmann NR, Gilliard AN, and Reitmayer AK (2017). Smart hydrogel sensor for detection of organophosphorus chemical warfare nerve agents. J. Macromol. Sci. A 54, 40-46.

12. Walker JP, and Asher SA (2005). Acetylcholinesterase-based organophosphate nerve agent sensing photonic crystal. Anal. Chem 77, 1596-1600. [PubMed: 15762562]

13. Qi F, Yan C, Meng Z, Li S, Xu J, Hu X, and Xue M (2019). Acetylcholinesterase functionalized two-dimensional photonic crystal for the sensing of G-series nerve agents. Anal. Bioanal. Chem 411, 2577-2585. [PubMed: 30847569]

14. Sun X, and Anslyn EV (2017). An auto-inductive cascade for the optical sensing of thiols in aqueous media: Application in the detection of a VX nerve agent mimic. Angew. Chem. Int. Ed. Engl 56, 9522-9526. [PubMed: 28586521]

15. Diehl KL, Kolesnichenko IV, Robotham SA, Bachman JL, Zhong Y, Brodbelt JS, and Anslyn EV (2016). Click and chemically triggered declick reactions through reversible amine and thiol coupling via a conjugate acceptor. Nat. Chem 8, 968-973.

16. Kim K, Tsay OG, Atwood DA, and Churchill DG (2011). Destruction and detection of chemical warfare agents. Chem. Rev 111, 5345-5403. [PubMed: 21667946]

17. Balasubramanian S, Kulandaisamy AJ, Babu KJ, Das A, and Rayappan JBB (2021). Metal organic framework functionalized textiles as protective clothing for the detection and detoxification of chemical warfare agents: a review. Ind. Eng. Chem. Res 60, 4218-4239. 
18. Climent E, Biyikal M, Gawlitza K, Dropa T, Urban M, Costero AM, Martinez-Manez R, and Rurack K (2017). Determination of the chemical warfare agents Sarin, Soman and Tabun in natural waters employing fluorescent hybrid silica materials. Sens. Actuators B Chem 246, 1056-1065.

19. Cavalcante SFD, Simas ABC, and Kuca K (2019). Nerve agents' surrogates: invaluable tools for development of acetylcholinesterase reactivators. Curr. Org. Chem 23, 1539-1559.

20. Bobbitt NS, Mendonca ML, Howarth AJ, Islamoglu T, Hupp JT, Farha OK, and Snurr RQ (2017). Metal-organic frameworks for the removal of toxic industrial chemicals and chemical warfare agents. Chem. Soc. Rev 46, 3357-3385. [PubMed: 28345694]

21. Sun X, Chwatko M, Lee DH, Bachman JL, Reuther JF, Lynd NA, and Anslyn EV (2020). Chemically triggered synthesis, remodeling, and degradation of soft materials. J. Am. Chem. Soc 142, 3913-3922. [PubMed: 32011873]

22. Ishibashi JSA, and Kalow JA (2018). Vitrimeric silicone elastomers enabled by dynamic Meldrum's acid-derived cross-links. ACS Macro Lett. 7, 482-486.

23. DeCollo TV, and Lees WJ (2001). Effects of aromatic thiols on thiol-disulfide interchange reactions that occur during protein folding. J. Org. Chem 66, 4244-4249. [PubMed: 11397160]

24. Ganesan K, Raza SK, and Vijayaraghavan R (2010). Chemical warfare agents. J. Pharm. Bioallied Sci 2, 166-178. [PubMed: 21829312]

25. Kucernak ARJ, Fahy KF, and Sundaram VNN (2016). Facile synthesis of palladium phosphide electrocatalysts and their activity for the hydrogen oxidation, hydrogen evolutions, oxygen reduction and formic acid oxidation reactions. Catal. Today 262, 48-56.

26. Gregory MJ, and Bruice TC (1967). Nucleophilic displacement reactions at the thiol ester bond. V. reactions of 2,2,2-trifluoroethyl thiolacetate. J. Am. Chem. Soc 89, 2121-2127.

27. Anslyn EV, and Dougherty DA (2006). Modern Physical Organic Chemistry (University Science Books), pp. 355-419.

28. Jiang W, Cao Y, Liu Y, and Wang W (2010). Rational design of a highly selective and sensitive fluorescent PET probe for discrimination of thiophenols and aliphatic thiols. Chem. Commun. (Camb.) 46, 1944-1946. [PubMed: 20198261]

29. Kumar V, and Rana H (2015). Chromogenic and fluorogenic detection and discrimination of nerve agents tabun and VX. Chem. Commun. (Camb.) 51, 16490-16493. [PubMed: 26394304]

30. Kumar V, Raviraju G, Rana H, Rao VK, and Gupta AK (2017). Highly selective and sensitive chromogenic detection of nerve agents (sarin, tabun and VX): a multianalyte detection approach. Chem. Commun. (Camb.) 53, 12954-12957. [PubMed: 29159359]

31. Kumar V, Rana H, Raviraju G, Garg P, Baghel A, and Gupta AK (2016). Chromogenic and fluorogenic multianalyte detection with a tuned receptor: refining selectivity for toxic anions and nerve agents. RSC Advances 6, 59648-59656.

32. Barba-Bon A, Costero AM, Gil S, Martínez-Máñez R, and Sancenón F (2014). Selective chromofluorogenic detection of DFP (a Sarin and Soman mimic) and DCNP (a Tabun mimic) with a unique probe based on a boron dipyrromethene (BODIPY) dye. Org. Biomol. Chem 12, 87458751. [PubMed: 25260024]

33. Dominguez MN, Howard MP, Maier JM, Valenzuela SA, Sherman ZM, Reuther JF, Reimnitz LC, Kang J, Cho SH, Gibbs SL, et al. (2020). Assembly of linked nanocrystal colloids by reversible covalent bonds. Chem. Mater 32, 10235-10245.

34. Boles MA, Ling D, Hyeon T, and Talapin DV (2016). The surface science of nanocrystals. Nat. Mater 15, 141-153. [PubMed: 26796733]

35. Dong A, Ye X, Chen J, Kang Y, Gordon T, Kikkawa JM, and Murray CB (2011). A generalized ligand-exchange strategy enabling sequential surface functionalization of colloidal nanocrystals. J. Am. Chem. Soc 133, 998-1006. [PubMed: 21175183] 


\section{Highlights}

A self-degradable hydrogel is used as a sensor for a nerve agent tabun surrogate A series of reactions occur with the tabun mimic to trigger the cascade

The hydrogel is used as a membrane to study the degradation rate

The membrane degradation is confirmed using nanocrystals 
A<smiles>CCOP(N)(=O)OCC</smiles><smiles>CSc1ccc(O)cc1</smiles>

6
TEA

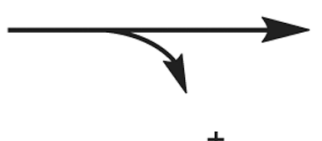

$-\mathrm{CN}, \mathrm{H}-\mathrm{NEt}_{3}$<smiles>CCOP(=O)(OCC)Oc1ccc(SC)cc1</smiles>

B<smiles>COc1ccc(SC(C)=O)cc1</smiles>

Figure 1. Model studies for rate constant

(A) Model reaction to release cyanide.

(B) Model reaction for cyanide capture and thiol release.

See also Table S1. 

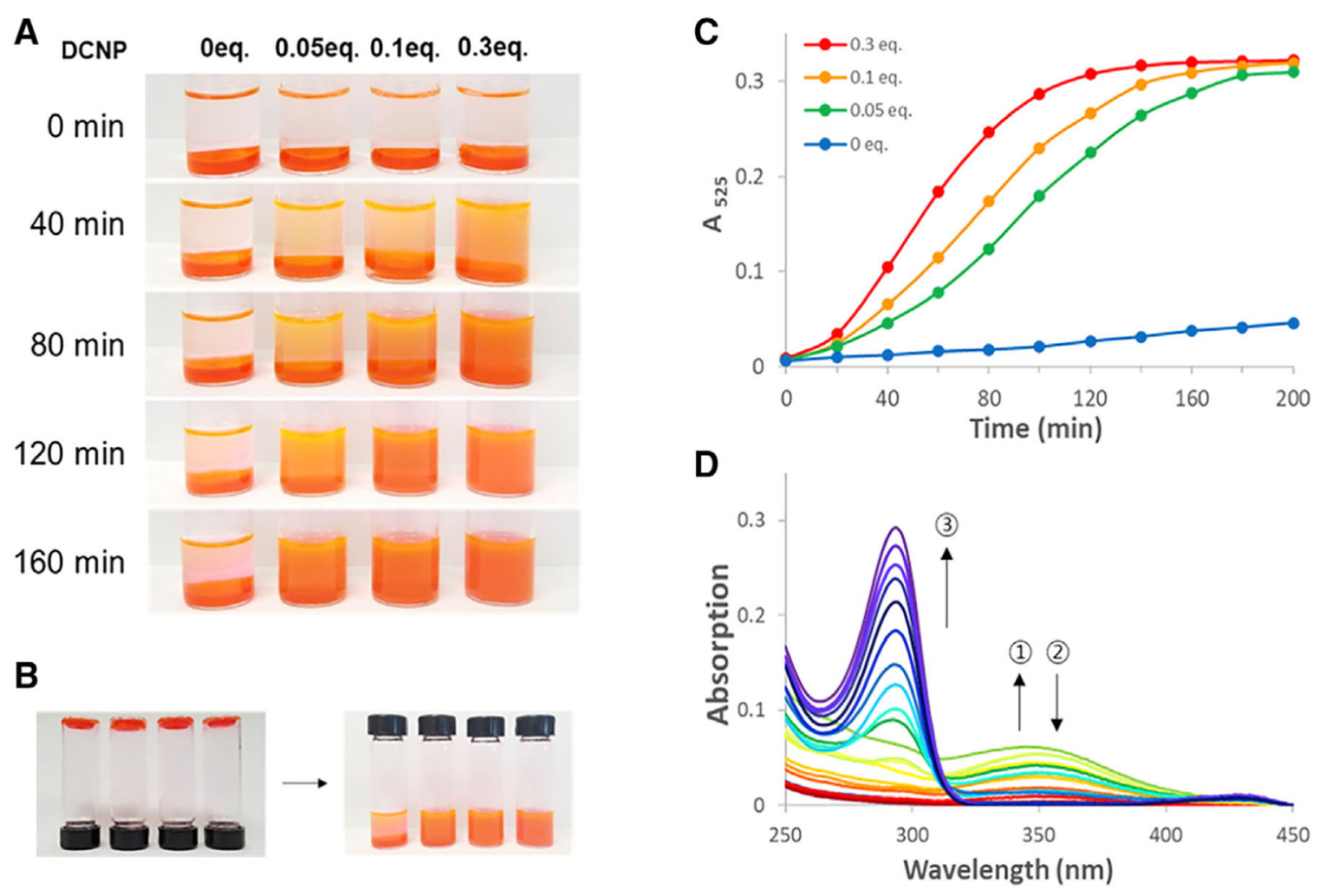

Figure 2. A titration study at physiological pH

A titration study was conducted at physiological $\mathrm{pH}$ (phosphate-buffered saline [PBS]).

After reacting $4(1.5 \mu \mathrm{mol})$, DCNP $(0,0.25,0.5$, and $1.5 \mu \mathrm{mol})$, and triethylamine $(3 \mu \mathrm{mol})$ in acetonitrile for $30 \mathrm{~min}$, the resulting mixture was added to $5 \mathrm{~mL}$ PBS buffer in the presence of BMEox $(5 \mu \mathrm{mol})$ and 3 with R6G. The reactions were then shaken.

(A) Photos of the four samples with different amount of DCNP.

(B) Photos before (gel) and after (sol) the test along with the arrow.

(C) Absorption data for the time-dependent release of R6G from the degradation of 3 at 525 $\mathrm{nm}$.

(D) Absorption spectra of kinetic data with $0.25 \mu \mathrm{mol}$ of DCNP. The spectra were changed according to the numbers and arrows.

See also Figure S8. 
A

Figure 3. Membrane degradation test of 3

There were four different weights and thicknesses of gels created with $\mathbf{3}$, which were synthesized with 5, 7, 9, and $11 \mu \mathrm{mol}$ of the Meldrum's acid linker in the gel.

(A) Thickness of 3: 1.7, 2.2, 2.6, and $3.2 \mathrm{~mm}$.

(B) A photo for the four samples before connecting the top vial.

(C) On the top between the two connected vials a mixture from the reaction of 4 (3 $\mu \mathrm{mol})$ with DCNP $(3 \mu \mathrm{mol})$, and triethylamine $(6 \mu \mathrm{mol})$ in acetonitrile $(30 \mathrm{~min})$ was added to PBS (15 $\mathrm{mL})$ buffer in the presence of R6G (1 mM).

See also Figure S13. 
A

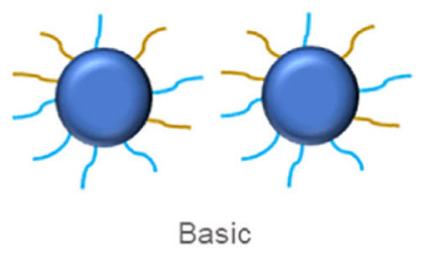

$\sqrt{\square}$

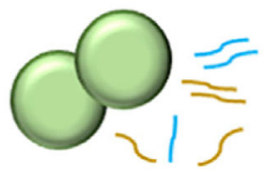

Acidic
B

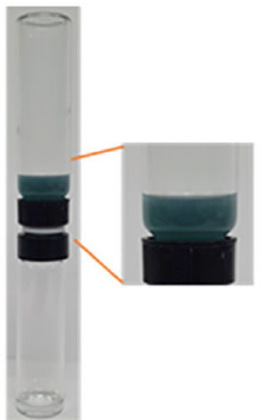

$\downarrow$

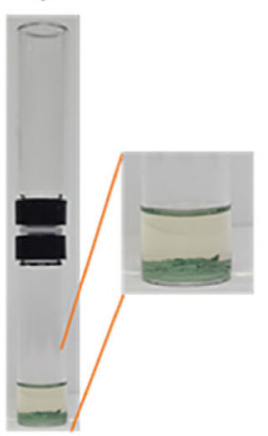

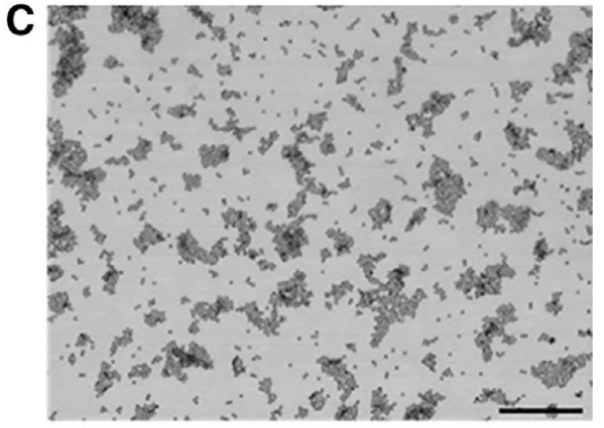

D

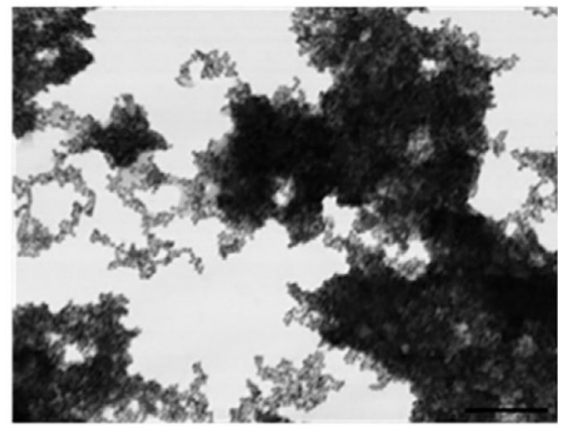

Figure 4. Experiment showing the alteration in degree of dispersion of nanocrystals due to a change in $\mathbf{p H}$ induced by membrane decomposition

(A) Representation of the ITO NCs with the ligands functionalized on the NC surface. Acidic conditions cause the ligands to be stripped from the NCs, causing NC degradation and aggregation.

(B) Top is a picture before the membrane degradation, and the bottom is a picture after degradation. The top vial contained a $\mathrm{pH} 10$ aqueous solution $(4 \mathrm{~mL})$ containing dispersed colloidal NCs, and the bottom was $\mathrm{pH} 3$ water $(1 \mathrm{~mL})$.

(C and D) STEM images before and after, respectively, membrane degradation. Both scale bars represent $250 \mathrm{~nm}$.

See also Figure S14. 


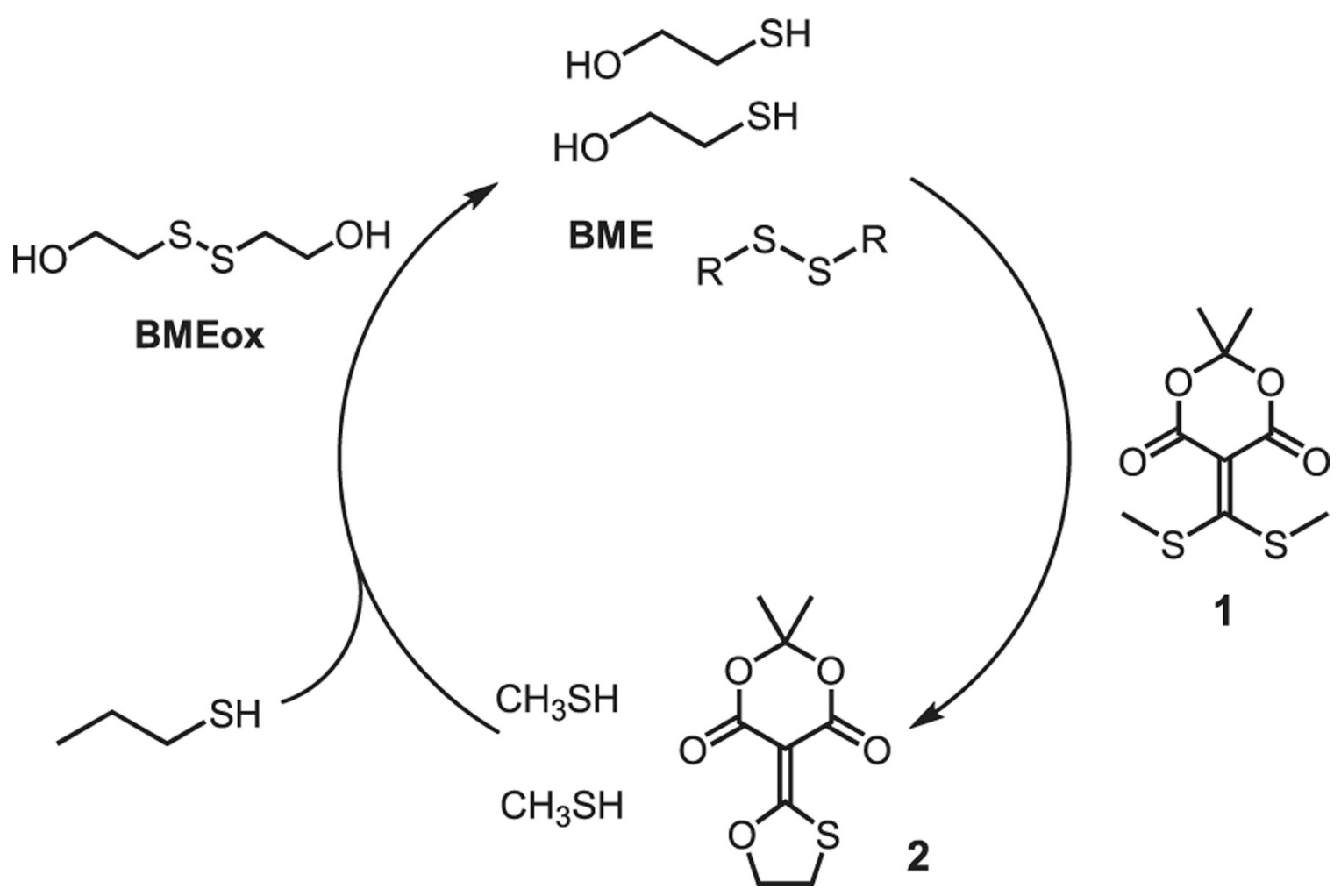

Scheme 1.

A thiol auto-inductive cascade in the previous work 
<smiles>CCOP(C)(=O)N(C)C</smiles>

Tabun<smiles>CCOP(=O)(C#N)OCC</smiles>

DCNP

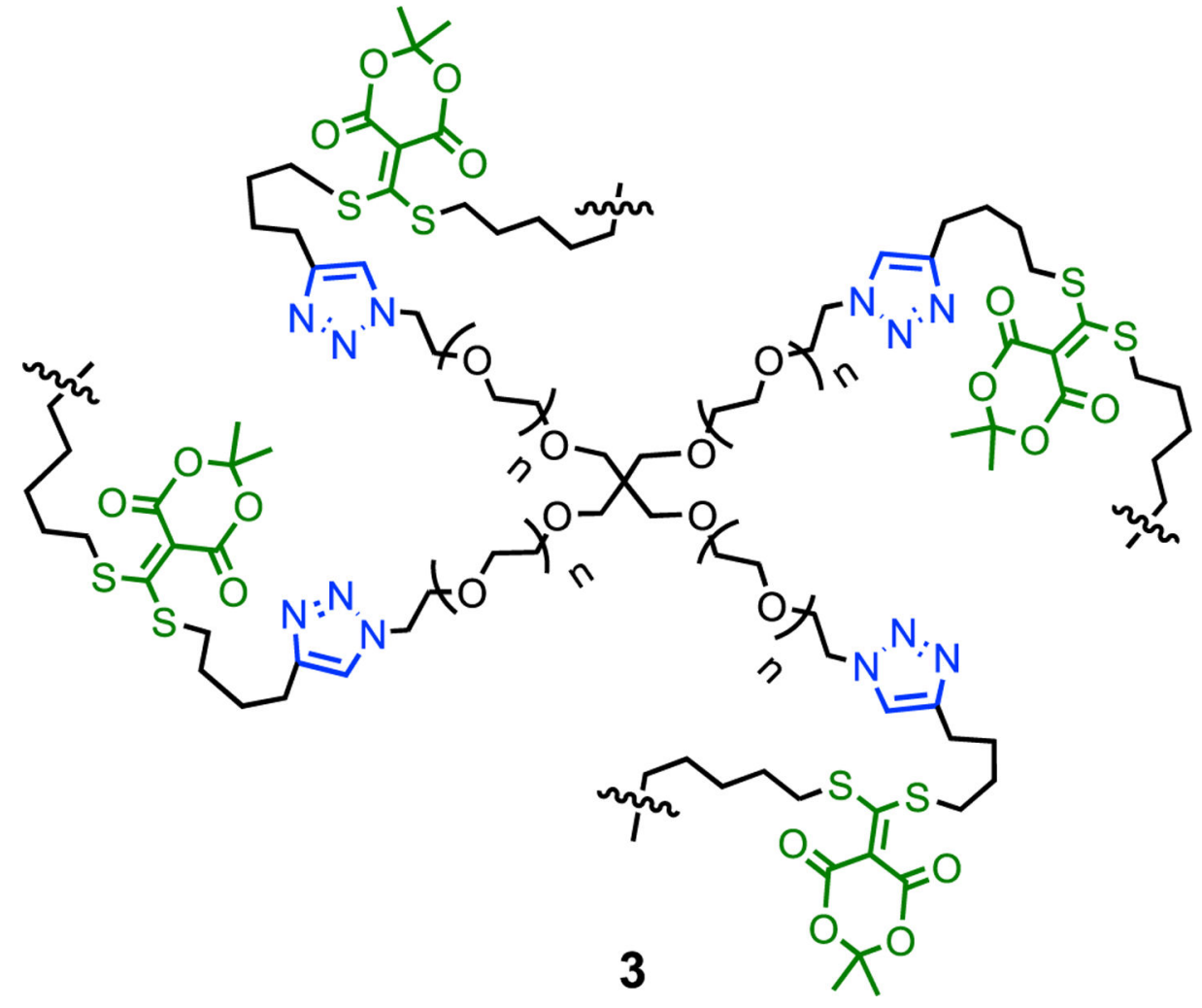

Scheme 2.

Selected tabun surrogate and synthesized hydrogel 


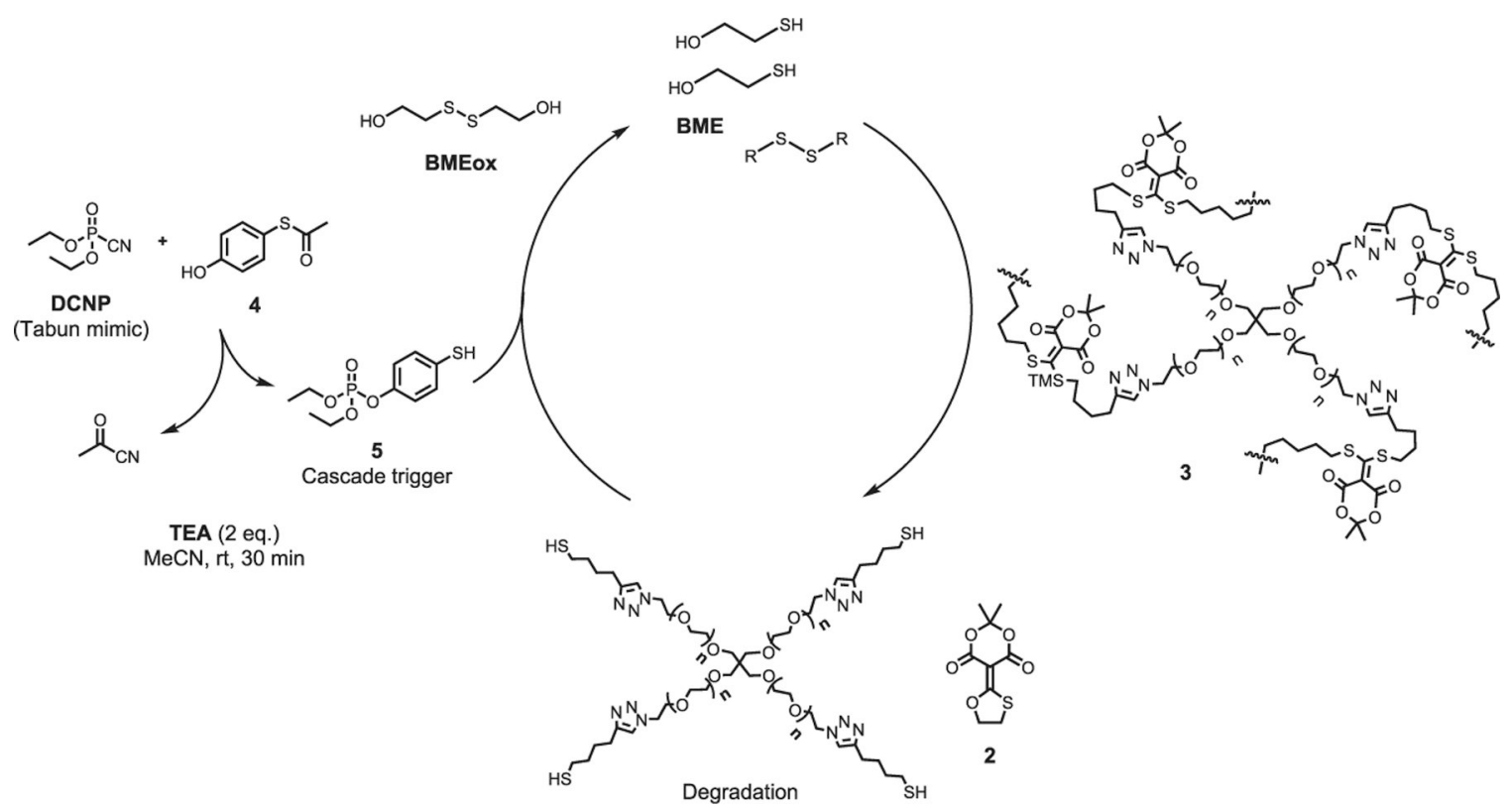

Scheme 3 .

Detection of a tabun surrogate via a self-propagating cascade with perturbation of gel formation 